\title{
Patches in a side-by-side configuration: a description of the flow and deposition fields.
}

\author{
D. Meire \\ Department of Civil Engineering, Hydraulics Laboratory \\ Ghent University, Ghent, Belgium \\ J. Kondziolka \& H.M. Nepf \\ Department of Civil and Environmental Engineering \\ Massachusetts Institute of Technology, Massachusetts, USA
}

\begin{abstract}
In the last few decades, a lot of research attention has been paid to flow-vegetation interactions. Starting with the description of the flow field around uniform macrophyte stands, research has evolved more recently to the description of flow fields around individual, distinct patches. However, in the field, vegetation patches almost never occur in isolation. As such, patches will influence each other during their development and interacting, complex flow fields can be expected.
\end{abstract}

In this study, two emergent patches of the same diameter $(D=22 \mathrm{~cm})$ and a solid volume fraction of $10 \%$ were placed in a side-by-side configuration in a lab flume. The patches were built as an array of wooden cylinders, and the distance between the patches (gap width $\Delta$ ) was varied between $\Delta=0$ and $14 \mathrm{~cm}$. Flow measurements were performed by a 3D Vectrino Velocimeter (Nortek AS) at mid-depth of the flow. Deposition experiments of suspended solids were performed for selected gap widths.

Directly behind each patch, the wake evolved in a manner identical to that of a single, isolated patch. On the centerline between the patches, the maximum velocity $U_{\max }$ was found to be independent of the gap width $\Delta$. However, the length over which this maximum velocity persists, the potential core $L_{j}$, increased linearly as the gap width increased. After the merging of the wakes, the centerline velocity reaches a minimum value $U_{\text {min }}$. The minimum centerline velocity decreased in magnitude as the gap width decreased. The velocity pattern within the wake is reflected in the deposition patterns. An erosion zone occurs on the centerline between the patches, where the velocity is elevated. Deposition occurs in the low velocity zones directly behind each patch and also downstream of the patches, along the centerline between the patches at the point of local velocity minimum. This downstream deposition zone, a result of the interaction of neighbouring patch wakes, may facilitate the establishment of new vegetation, which may eventually inhibit flow between the upstream patches and facilitate patch merger.

\section{INTRODUCTION}

Hydraulic studies have mainly focused on long, uniform meadows, characterizing the bulk flow resistance (Kouwen and Unny 1975, Stephan and Gutknecht 2002, Jarvela 2005) and describing the vertical flow structure and turbulence characteristics, e.g. reviewed in Nepf (2012). However, vegetation is often found in patches of finite length and width (Naden et al. 2006, Temmerman et al. 2007, Schoelynck et al. 2012), rather than in continuous segments. As such, recent attention has been focused on the study of finite patches of vegetation, both in the lab and in the field (Cotton et al. 2006, Bouma et al. 2009, Zong and Nepf 2011). The interaction between neighboring patches has also been considered (Vandenbruwaene et al. 2011).

Rietkerk and Van de Koppel (2008) explained the process called spatial self-organization, in which large-scale, ordered spatial patterns occur because of feedbacks between small-scale landscape elements. Such landscape evolution has been demonstrated for a wide variety of ecosystems, such as mussel beds (van de Koppel et al. 2005), vegetation on tidal flats (van Wesenbeeck et al. 2008), and vegetation in 
lowland rivers (Schoelynck et al. 2012). In each case, the introduction of an organism produces positive feedbacks (stress reduction, accumulation of nutrients) and negative feedbacks (stress enhancement, depletion of nutrients), which influence the pattern of growth. For example, Bouma et al. (2009) show for intertidal macrophytes (Spartina anglica) that, above a certain threshold of vegetation density, sediment is trapped within the vegetation (positive feedback) and erosion is observed next to the vegetation (negative feedback).

Where macrophytes can establish, they will influence sediment transport and thus the bathymetry, and these biogeomorphic feedbacks are important to the further macrophyte development. Sites of erosion are places of lower nutrient availability that lead to less favorable conditions for plant growth (van Wesenbeeck et al. 2008). Sites of deposition, in contrast, are where seeds and organic matter will tend to accumulate, leading to favorable conditions for plant growth (Gurnell et al. 2005). Deposition of fine sediments in flow influenced by vegetation has been related to the characteristics of the mean and turbulent velocity field through laboratory studies (Chen et al. 2012) and field studies (Sand-Jensen 1998, Cotton et al. 2006, Schoelynck et al. 2012). Chen et al. (2012) modelled patches of emergent vegetation in a laboratory flume. They found that net deposition was generally inhibited in areas of high turbulent kinetic energy (TKE) or high velocity, likely due to resuspension, and generally enhanced in areas of low $T K E$ and low velocity. In particular, a region of low velocity and low $T K E$ occurred directly behind the patch over a length-scale of several patch diameters, and enhanced deposition was observed within this region. This is consistent with several field studies.

The current work builds on the previous studies of individual patches to consider the interaction between two adjacent patches. We explore how the spacing between patches influences the pattern of flow distribution and deposition in the wakes of the two patches.

\subsection{Flow adjustment to a single patch}

First, the main parameters used to describe flow to a single patch and the main characteristics of flow past an isolated patch are reviewed. For a given stem density, $n\left(1 / \mathrm{cm}^{2}\right)$, and mean stem diameter, $d(\mathrm{~cm})$, the frontal area per unit volume is $a=n \cdot d(1 / \mathrm{cm}) . D$ is the patch diameter $(\mathrm{cm})$. As the fluid passes around and through the patch, a shear layer forms between the slower-moving fluid behind the patch $\left(U_{1}\right)$ and the faster-moving fluid outside the patch wake $\left(U_{2}\right)$. These shear-layers, formed on either side of the patch, meet at the patch centerline at a distance $L_{1}$ from the patch. Over the distance $L_{1}$ on the patch centerline, the velocity $U_{1}$ remains unchanged. Beyond this point $\left(x>D+L_{1}\right)$, the wake velocity on the patch centerline, $U_{1}$, starts to increase. $U_{1}$ may be predicted from the non-dimensional flow blockage, $C_{D} a D$, where $C_{D}$ [-] is the drag coefficient for the stems within the patch (Chen et al. 2012).

\subsection{Flow adjustment to a pair of obstructions}

Flow and deposition patterns near a side-by-side pair of similar model vegetation patches is considered here. For side-by-side solid circular cylinders of diameter $D$, the wake characteristics depend on the distance between the two cylinders and the Reynolds number $\left(R e_{D}=U_{\infty} D / \nu\right)$, where $\nu\left(\mathrm{cm}^{2} / \mathrm{s}\right)$ is the kinematic viscosity (Sumner 2010). Three types of flow behavior are summarized by Sumner (2010). At separation distances less than $(0.10-0.20) D$, the two cylinders behave as a single bluff-body, as indicated by the formation of a single von Kármán vortex street, scaling with the total width across both cylinders, and thus having a lower frequency of vortex shedding compared to an individual cylinder. The flow between the two cylinders behaves as bleed flow, which lengthens the streamwise extent of the vortex formation region (Sumner et al. 1999). As the cylinders are moved further apart, $\Delta$ larger than 0.2 $D$ and less than $1.2 D$, a biased flow pattern develops in which flow through the gap is deflected toward one of the cylinders. The deflection angle of the gap flow increases as $\Delta / D$ decreases. The cylinder towards which the flow is deflected has a narrower and shorter near-wake zone and higher frequency shedding than the neighbouring cylinder. Finally, when the distance between the two cylinders $\Delta$ is larger than about 1.2 $D$, parallel vortex streets are observed, predominantly in anti-phase (Sumner et al. 1999, Sumner 2010).

The interaction between porous cylinders (a model for vegetation patches) has not been characterized as thoroughly as the interaction of solid cylinders. Vandenbruwaene et al. (2011) considered the change in flow distribution close to a pair of vegetation patches, to understand under what conditions adjacent patches would merge together, rather than remain separated by a channel. Their velocity measurements were taken adjacent to and in between patches of different diameters $(D)$ and different separation distances $(\Delta)$. Acceleration of flow, i.e elevated velocity, between the patches was observed for all conditions, however the acceleration decreased, compared to the acceleration at the outer edges of the patches, below a gap width $\Delta / D \approx 0.1$. From these observations alone, one might conclude that adjacent patches cannot merge, since flow acceleration, which would tend to promote erosion and inhibit plant growth, will always be maintained in the space between the patches. However, we 
hypothesize that a different conclusion might be reached if we consider the flow development in the wake of the patches. As described above, the wake behind a single patch is a region of sediment deposition and potential vegetation growth. Based on the solid-cylinder literature (above) we anticipate that for some interpatch distances, a merged wake may form behind the pair of patches that resembles the wake of a larger, single patch. Deposition and vegetation growth within the merged wake could eventually influence the flow distribution between the upstream patches and allow for patch merger. Motivated by this hypothesis, the focus in this study is to characterize the flow and the deposition pattern in the wake behind a pair of side-by-side patches, as a function of the interpatch distance.

\section{MATERIAL AND METHODS}

Flow and deposition measurements were performed in a recirculating flume $16-\mathrm{m}$ long and $1.2-\mathrm{m}$ wide. The discharge was set by a variable-speed pump to produce a depth-averaged upstream velocity $U_{\infty}$ of approximately $10 \mathrm{~cm} / \mathrm{s}$. A downstream, adjustable weir was used to set a flow depth $H$ of $14 \mathrm{~cm}$.

Table 1: Summary of measurements. $D$ is the diameter of the patch, $d$ is the cylinder diameter, $a$ is the frontal area per unit volume, $\phi$ is the solid volume fraction of the patch, $U_{\infty}$ is the upstream, depth-averaged velocity and $\Delta$ is the gap distance between the patches.

\begin{tabular}{llc}
\hline$D$ & $(\mathrm{~cm})$ & $22 \pm 0.8$ \\
$d$ & $(\mathrm{~mm})$ & $3.2 \pm 0.1$ \\
$a$ & $(1 / \mathrm{cm})$ & $0.40 \pm 0.01$ \\
$a D$ & $(-)$ & $8.8 \pm 0.5$ \\
$\phi$ & $(\%)$ & $10 \pm 0.5$ \\
Patch Type & & Dense \\
$U_{\infty}$ & $(\mathrm{cm} / \mathrm{s})$ & $9.4 \pm 0.3$ \\
$\Delta$ & $(\mathrm{cm})$ & $0,2,5,8,11,14$ \\
Deposition & & $\Delta=0,2,11 \mathrm{~cm}$ \\
\hline
\end{tabular}

The circular patches were constructed from wooden dowels and extended through the water surface, a mimicry for real, emergent vegetation, such as reeds. The dowels had a diameter of $d=3.2$ $\mathrm{mm}$, a height of $16 \mathrm{~cm}$ and were held in perforated PVC boards. Experimental runs were made for one patch diameter of vegetation $(D=22 \mathrm{~cm})$ at high flow blockage, defined as $C_{D} a D>4$ (Chen et al. 2012). The patch density for this case was $a \approx 0.4$ $\mathrm{cm}^{-1}$, which corresponds to a solid volume fraction of $\phi=(\pi / 4) a d \approx 10 \%$, and a $C_{D} a D$ of 8.8 , taking $C_{D}=1$. The distance between the patches $\Delta(\mathrm{cm})$ was varied by placing PVC strips of variable width in between the patch boards. The distance $\Delta$ was varied from $\Delta / D=0$ to a maximum of $\Delta / D=1$. The patches were placed in a side-by-side configuration at $7 \mathrm{~m}$ from the flume entrance. An overview on the tests is given in Table 1.

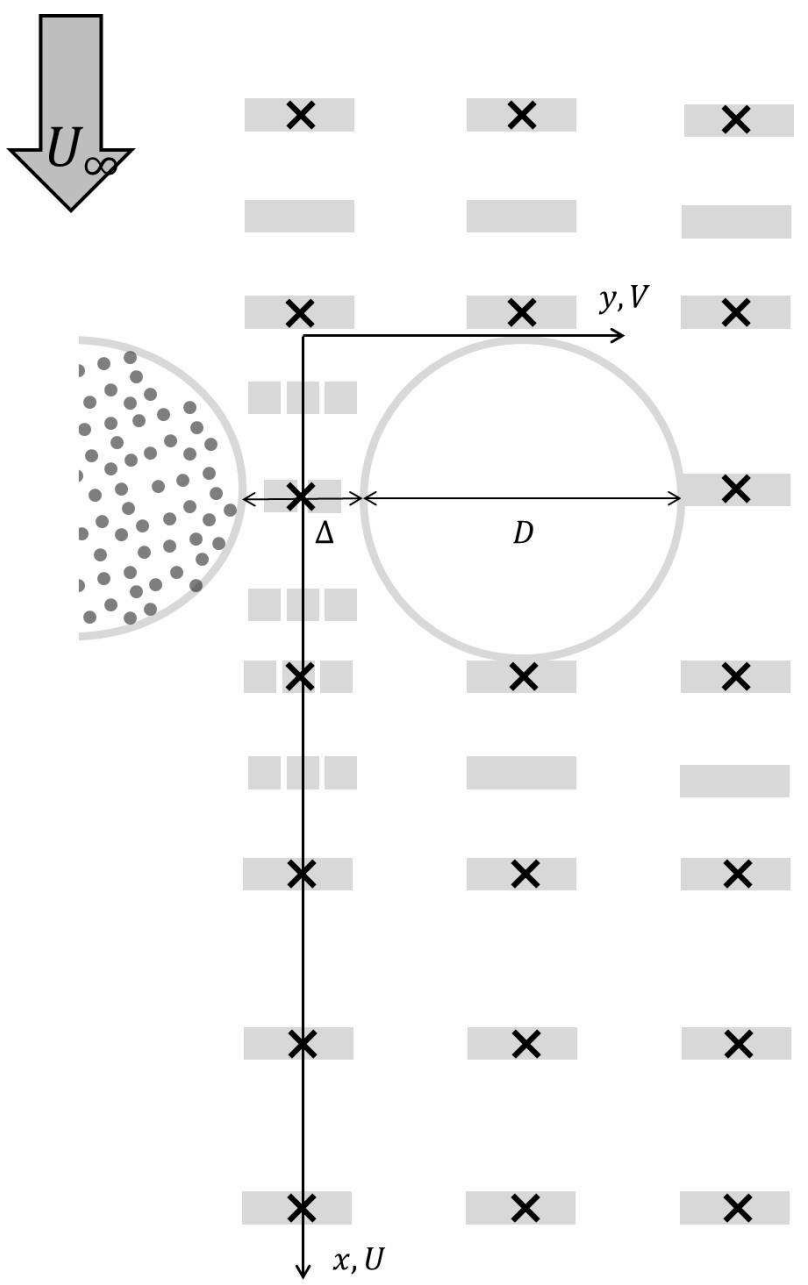

Figure 1: Schematic top-view of flume close to the model vegetation patches (circles), not to scale. The coordinate axis in the horizontal plane is shown, with velocities $U$ and $V$ in the directions of $x$ and $y$, respectively, with $x=0$ at the patch leading edge and $y=0$ on the centerline between the patches. The vertical axis is upwards (not depicted). Two patches, seperated by a gap $\Delta$, consist of staggered arrays of dowels. The positions of the velocity measurements are indicated by heavy crosses, the positions of the deposition slides are indicated by gray rectangles. Only measurements close to the patch are shown. 


\subsection{Velocity measurements}

A 3D Vectrino (Vectrino Velocimeter, Nortek AS), which measures velocities using the acoustic Doppler technique, was used for velocity measurements. The sampling volume of the ADV was located at mid-depth, and velocity time-series for a period of at least $240 \mathrm{~s}$ at a rate of $25 \mathrm{~Hz}$ were obtained. These data-series were processed in MATLAB, to filter data points that had especially low values in signal to noise ratio $(\mathrm{SNR}<15)$, correlation $($ corr $<70)$ or amplitude (amp < 90) (McLelland and Nicholas 2000). The mean time-averaged velocities, respectively $(U, V, W)$ for the $(x, y, z)$ directions, were taken as the average of the remaining measurements over the recording period. Fluctuations around the mean, denoted $u^{\prime}, v^{\prime}, w^{\prime}$, were taken by subtracting the mean velocity from each instantaneous record.

The coordinate system is defined with the streamwise coordinate $x=0$ at the upstream edge of the patches and the lateral coordinate $y=0$ at the center of the flume, which is also the center of the gap between the patches, as shown in Figure 1. Measurements were made from $2.2 \mathrm{~m}$ upstream to $5 \mathrm{~m}$ downstream of the patches. The measurement positions were spaced more tightly close to the patches.

\subsection{Deposition Experiments}

Conditions were chosen for the deposition experiments to mimic the transport of organic matter and fine sediment, which produce substrate high in nutrient content and favorable to plant growth. The deposition experiments were carried out with a model sediment that was scaled to provide a desired ratio of settling velocity $V_{s}$ to open-channel bed friction velocity. In the experiments $u^{*}=0.7 \mathrm{~cm} / \mathrm{s}$, estimated from the bed drag coefficient $\left(C_{f}=0.006\right.$, White and Nepf (2007)). $10 \mu \mathrm{m}$ glass sphere particles (Potters Industry, Valley Forge, PA) were selected with a settling velocity $V_{s}=0.01 \mathrm{~cm} / \mathrm{s}$, so that $V_{s} / u^{*}=$ 0.014 , which is within the range expected in the field $\left(V_{s} / u^{*}=0.002\right.$ to 0.3 , see discussion in Ortiz and Nepf (2014)). In addition, the conditions are similar to a previous study (Zong and Nepf (2010)), in which clear differences in deposition were observed between the open channel and vegetated regions of the channel.

Deposition experiments were performed for three different configurations, with a relative gap distance $\Delta / D$ of $0.5,0.1$ and 0 . Three repetitions were performed for each set of conditions. A control experiment with no patches in the flume was also performed. Before starting the experiment, the flume was drained and cleaned. Glass microscope slides (VWR VistaVision Microscope Slides) with a thickness of $1 \mathrm{~mm}$ and an area of $7.5 \times 2.5 \mathrm{~cm}$ or
$2.5 \times 2.5 \mathrm{~cm}$ were thoroughly washed, dried in an oven at 70 degrees Celsius for 4 hours, labeled, and then weighed. Slides were placed in 5 longitudinal profiles, partially shown in Figure 1: on the centerline of the gap between the two patches $(y=0)$, on the centerline of each patch $(y= \pm(D+\Delta) / 2)$, and on the outside edge of each patch $(y= \pm D+\Delta / 2)$. The spacing between the slides in the streamwise direction is small $(5 \mathrm{~cm})$ close to the patch and increased further to a maximum spacing of $30 \mathrm{~cm}$. At the start of the experiment, $600 \mathrm{~g}$ of sediment was poured into the tailbox of the flume. Based on visual observation, the particles were mixed over the flow depth directly after entering the flume and a uniform condition over the flume length was observed within 2 minutes. The particles were recirculated in the flume for 4 hours. At the end of the experimental run, the flow was slowly decelerated to avoid waves, the flume was drained, and then the flume was left to dry for at least 2 days. The slides were baked at 90 degrees Celsius to remove additional moisture, and weighed after the experiment. The weight difference before and after the experiment is defined as the net deposition $\left(\mathrm{g} / \mathrm{cm}^{2}\right)$.

The net deposition mean $\left(\mu_{r i}\right)$ and standard error $\left(S E_{r i}\right)$ of each point were computed using the three replicates for each experimental configuration. To isolate deviations from the mean channel deposition, the mean of each experiment $\left(\mu_{r}\right)$ was subtracted from each individual data point. The standard error for the samples in the control experiment $\left(S E_{c}\right)$ was also computed. We considered a point to have enhanced net deposition, relative to the control, if the net deposition differed from the experiment mean by more than the sum of the standard errors:

$\mu_{r i}-\mu_{r}>S E_{r i}+S E_{c}$

\section{RESULTS}

\subsection{Velocity transects}

The mean, streamwise velocity in the center of the gap between the patches and through the center of the patches is shown in Figure 2 for gap widths $\Delta / D$ $=0.1$ to 0.5 . For the longitudinal profiles through the patch centers, no difference could be observed between the patches (the left patch is indicated with the symbol $\Delta$ in Figure 2, the right patch with + ). The only exception is found for the case $\Delta=0 \mathrm{~cm}$, where a strong asymmetry was observed between the two patch wakes (data not shown). Furthermore, no fundamental differences between the wakes of side-by-side patches and the wake behind an isolated patch could be observed. For each of the patches in the pair, $U_{1} / U_{\infty}$ was between 0.02 and 0.05 , agreeing within uncertainty with the value of 0.03 , found for isolated patches of a similar flow blockage 
Table 2: Parameters describing the velocity evolution on the centerline between the patches at different gap distances. $L_{0}$ is the upstream adjustment length, $U_{\max }$ is the maximum centerline velocity, $U_{\infty}(9.4 \pm 0.3 \mathrm{~cm} / \mathrm{s})$ is the far upstream incoming velocity, $U_{\min }$ is the minimum centerline velocity, $L_{m}$ is the distance from the trailing edge of the patches to the point where the centerline velocity reaches $U_{\min } . L_{S D}$ is the distance from the trailing edge of the patches to the start of secondary deposition zone.

\begin{tabular}{lcccccc}
\hline \hline Case & $L_{0}(\mathrm{~cm})$ & $U_{\max } / U_{\infty}$ & $U_{\min } / U_{\infty}$ & $L_{j}(\mathrm{~cm})$ & $L_{m}(\mathrm{~cm})$ & $L_{S D}(\mathrm{~cm})$ \\
\hline Dense, $\Delta=0$ & $44 \pm 5$ & $1.14 \pm 0.2$ & $0.07 \pm 0.01$ & - & $87 \pm 6$ & $44 \pm 6$ \\
Dense, $\Delta=2$ & $41 \pm 5$ & $1.64 \pm 0.09$ & $0.25 \pm 0.02$ & $5 \pm 4$ & $75 \pm 4$ & $105 \pm 6$ \\
Dense, $\Delta=5$ & $42 \pm 5$ & $1.67 \pm 0.06$ & $0.42 \pm 0.03$ & $17 \pm 3$ & $111 \pm 5$ & - \\
Dense, $\Delta=8$ & $37 \pm 5$ & $1.66 \pm 0.07$ & $0.56 \pm 0.03$ & $28 \pm 4$ & $134 \pm 6$ & - \\
Dense, $\Delta=11$ & $36 \pm 5$ & $1.66 \pm 0.06$ & $0.60 \pm 0.04$ & $30 \pm 4$ & $135 \pm 6$ & $155 \pm 7$ \\
Dense, $\Delta=14$ & $37 \pm 5$ & $1.65 \pm 0.06$ & $0.67 \pm 0.03$ & $36 \pm 5$ & $149 \pm 7$ & - \\
\hline
\end{tabular}
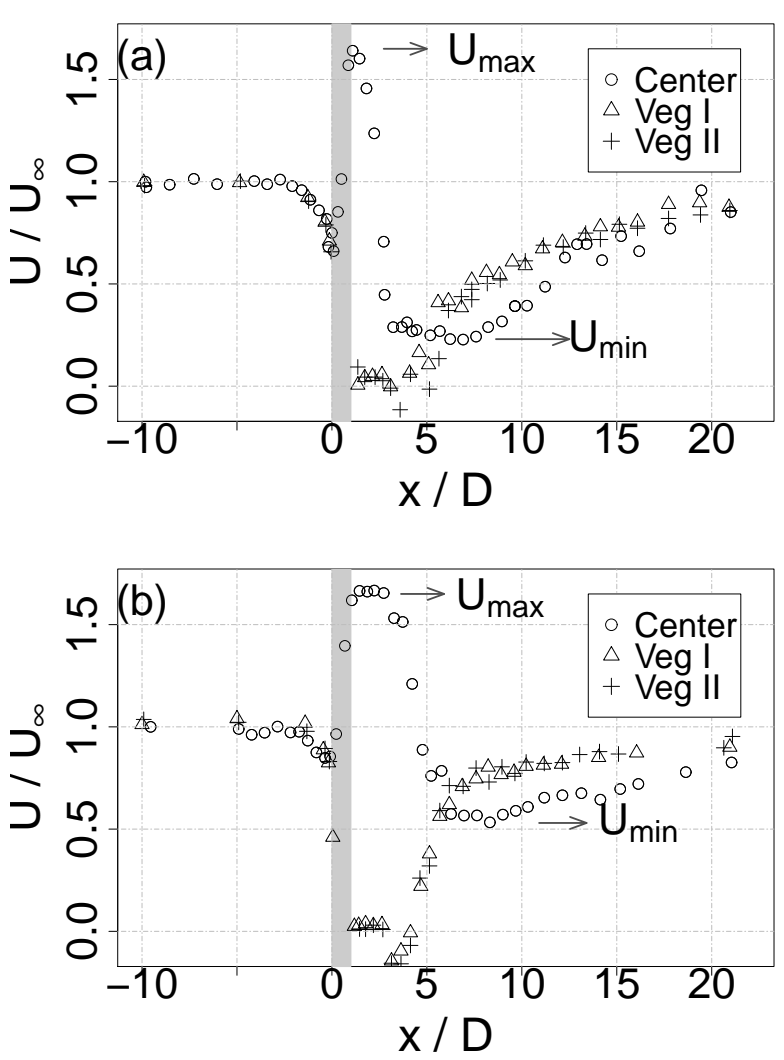

Figure 2: Mean, streamwise velocity on the flume centerline (center of the gap) and on two vegetation centerlines for two gap spacings: (a) $\Delta=2 \mathrm{~cm}$ and (b) $\Delta=11 \mathrm{~cm}$

(Chen et al. 2012). Chen et al. (2012) proposed a steady wake length of $L_{1}=2.5( \pm 0.4) D$ for single patches with high flow blockage $\left(C_{D} a D>4\right)$. In the side-by-side configuration, it is found that $L_{1}=2.4$ $( \pm 0.1) D$.

The mean, streamwise velocity along the centerline between the patches (Figure 2 and parameters in Table 2) for every gap spacing is essentially identical upstream of the patches, with deceleration beginning about $L_{0}=2 D$ upstream. Deviation between gap width conditions begins $1 D$ upstream of the patches, with the velocity reduction more pronounced for smaller $\Delta$, with a maximum reduction of $40 \%$ for $\Delta$ $=0$.

Between the patches, the flow accelerates and reaches a maximum $\left(U_{\max }\right)$ directly behind the patches $(x / D=1)$ indicated in Fig 2. Surprisingly, the maximum velocity is independent of the gap spacing and its value can be predicted by a simple conservation of mass over the flume width within an accuracy of $10 \%$. The maximum centerline velocity is sustained over a distance $L_{j}$. The flow which exits the gap can be considered similar to a turbulent jet, for which this region of constant, maximal velocity $\left(L_{j}\right)$ is called the potential core (Lee \& Chu 2003). A linear relation between the length of the potential core $L_{j}$ and the gap width $\Delta$ was observed (eq. 2), with a slope value of 2.8, close to the typical ratios of 3 to 6 (Lee and Chu 2003).

$$
L_{j}=2.8( \pm 0.2) \Delta \quad\left(R^{2}=0.91\right)
$$

The deceleration is followed by a sustained region of minimum velocity $\left(U_{m i n}\right.$, indicated in Figure 2$)$. The magnitude of $U_{\min }$ can be predicted from a simple model that accounts for the mixing of the jet with the lower velocity fluid in the wakes to either side of the jet. The lowest centerline velocity will occur just as the fluid at each wake centerline (the lowest wake velocity) is blended with the jet. This occurs when the blending distance, $W_{m}$, extends between the two wake centerlines, $W_{m}=D / 2+\Delta+D / 2=D+\Delta$. A simple conservation of mass over this width results in equation 3, where $\epsilon$ is an offset value, which accounts for the fact that the flow in between the patches doesn't go to 0 when the gap distance goes to zero.

$\frac{U_{\text {min }}}{U_{\infty}}=\frac{U_{\max }((\epsilon+\Delta) / D)+U_{1}}{U_{\infty}(1+((\epsilon+\Delta) / D))}$

A value of $\epsilon=1.8( \pm 0.4) \mathrm{cm}$ is found by fitting (3) to observed values of $U_{\min }$ using a nonlinear, least-square estimate employing a Gauss-Newton algorithm. The parameter $\epsilon$ is likely to be dependent on the shape, density, and homogeneity of the patches. $L_{m}$ is the distance from the trailing edge of the patches $(x=D)$ to the point where the decelerating jet reaches its minimal velocity $\left(U_{\min }\right) . L_{m}$ increases with gap width (data in Table 2). Physically, $L_{m}$ represents the point at which the two individual patch wakes merge to form a single, larger wake. Finally, when the shear layers formed at the outermost edges of the patch-pair grow to the centerline, the centerline velocity begins to increase. 


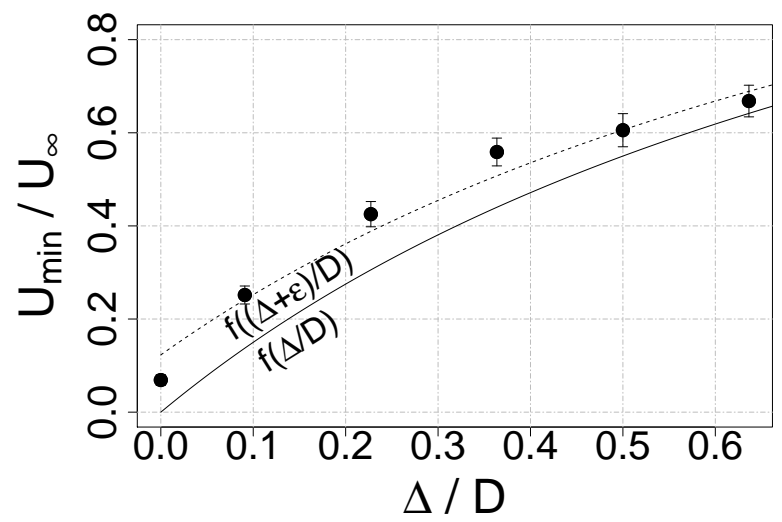

Figure 3: Average minimum, streamwise velocity on the centerline between patches for different gap spacings. The solid line is a fit with $\epsilon=0$, for the dashed line, $\epsilon=1.8 \mathrm{~cm}$

\subsection{Deposition}

The main characteristics of the velocity field are connected to the patterns of deposition. Under control conditions, with no patches in the flume, deposition was uniformly distributed (within a variation of $10 \%$ ) and specifically showed no tendency in the streamwise direction, indicating that the deposition was not supply limited (data not shown). With the patches in the flume, distinct patterns of deposition were observed. Deposition results are shown for the cases with a gap spacing of $\Delta / D=0.1$ and 0.5 in Figure 4 and 5, respectively.

Directly upstream of the patch pair, deposition was enhanced over a distance comparable to the upstream flow adjustment $\left(L_{0} \approx 2 D\right)$. Gurnell et al. (2001) and Zong and Nepf (2010) also observed enhanced deposition upstream of a patch, which was attributed to diminished local bed stress due to flow decelaration approaching the patch. Downstream of the patch pairs, three key features can be identified: a zone of enhanced deposition immediately behind each patch (Figure 5), a zone of reduced deposition in between the patches (Figure 4), and a secondary zone of enhanced deposition on the centerline between the patch pair and on the vegetation transect (Figure 4 and 5). The zone of reduced deposition between the patches is longer than the potential core in the jet region $L_{j}$, which can be explained by the fact that $T K E$ peaks in the decelaration zone (not shown) and the velocity remains elevated above the control $U_{\infty}$ for distances longer than $L_{j}$. A zone of secondary deposition on the centerline is a unique feature of the interaction between the two patch wakes. This second zone of deposition extends laterally over the width of the two patches and gap $(\Delta+2 D)$. The leading edge of this zone moves closer to the patches as the gap decreases (parameter $L_{S D}$ in Table 2), as can also be seen in Figure 4 for a gap of 2 and $11 \mathrm{~cm}$. At $\Delta / D$ $=0$, the secondary zone merges with the deposition zone of the individual patches.
We caution that the results presented here are for a single sediment size, concentration, and flow field. While suggestive of possible deposition patterns, the observed patterns may not be representative of all systems. For example, if the mean velocity is below the threshold for particle motion, a further depression of the velocity in the patch wakes may not lead to enhanced deposition. Similarly, different settling velocities of the sediment (associated with the $d_{50}$ of the sediment) may result in different extents and intensities of the deposition zones.

\section{DISCUSSION}

Our measurements have shown that the velocity and deposition patterns that occur directly behind individual patches are not significantly altered by patches in a side-by-side configuration. Specifically, directly behind each patch there is enhanced deposition that corresponds to a region of diminished mean velocity and turbulence, that extends a distance $L_{1}$ behind the patch. Beyond $L_{1}$, however, a neighboring patch can influence the velocity and deposition patterns. In particular, wake merger can produce a velocity minimum on the centerline between the patches at a distance $L_{m}$ downstream from the patches. The distance $L_{m}$ is a linear function of gap width. We observe that the velocity minimum produces a region of enhanced deposition (Figure 5). The location of this secondary deposition zone $\left(L_{S D}\right)$ increases with gap width. This secondary region of deposition may provide a positive feedback that eventually allows the two patches to merge. If this secondary zone of enhanced deposition facilitates the establishment and growth of vegetation, it will provide additional drag and flow blockage on the centerline between the original patches, which could reduce or completely halt the flow between the patches, setting up flow conditions that would allow for patch merger and lateral vegetation growth. Previous descriptions of vegetation-flow feedbacks identified positive feedbacks only for streamwise patch growth (Bouma et al. 2009) and negative feedbacks for lateral growth. By considering the interaction between neighboring patches we have identified a new, positive feedback for lateral growth.

\section{ACKNOWLEDGMENTS}

This material is based upon work supported by the National Science Foundation under Grant No. OCE 0751358 and EAR 0738352. Any opinions, findings, and conclusions or recommendations expressed in the material are those of the author(s) and do not necessarily reflect the views of the National Science Foundation. Dieter Meire wants to thank BOF (Bijzonder Onderzoeksfonds, B/10677/02 - BOF09/DOC/353) for personal research funding and the support of the 


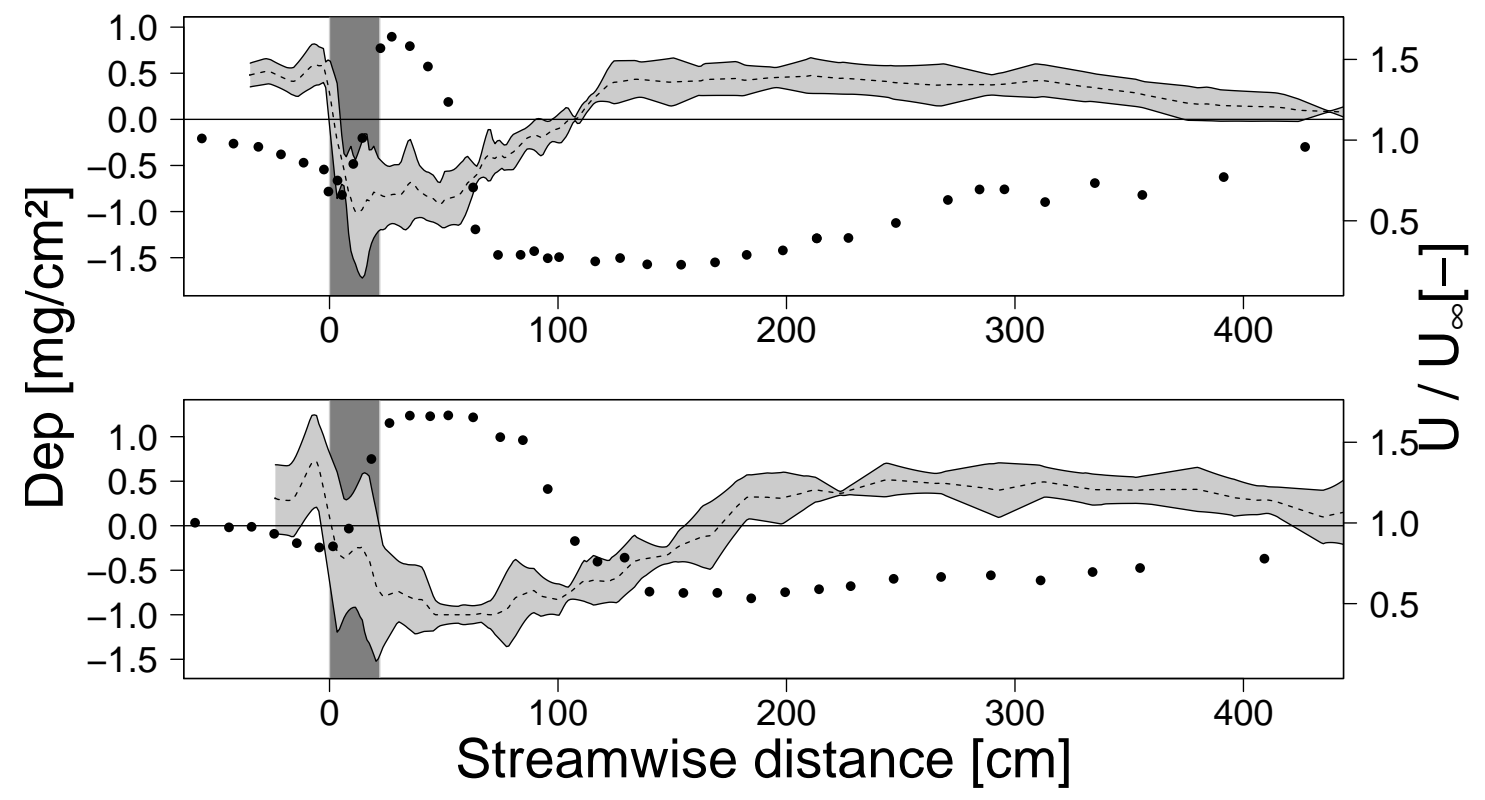

Figure 4: Average deposition $\left[\mathrm{mg} / \mathrm{cm}^{2}\right]$ from three measurements substracted from the mean deposition of each experimental run is shown in light gray, the interval indicating the error on the measurements. Mean, streamwise velocity $U$ on the centerline of the gap, normalized by the upstream velocity $U_{\infty}$ for a gap spacing of $2 \mathrm{~cm}$ (above) and $11 \mathrm{~cm}$ (below) is indicated by solid, black dots. The position of the patch is indicated by a dark, grey bar.

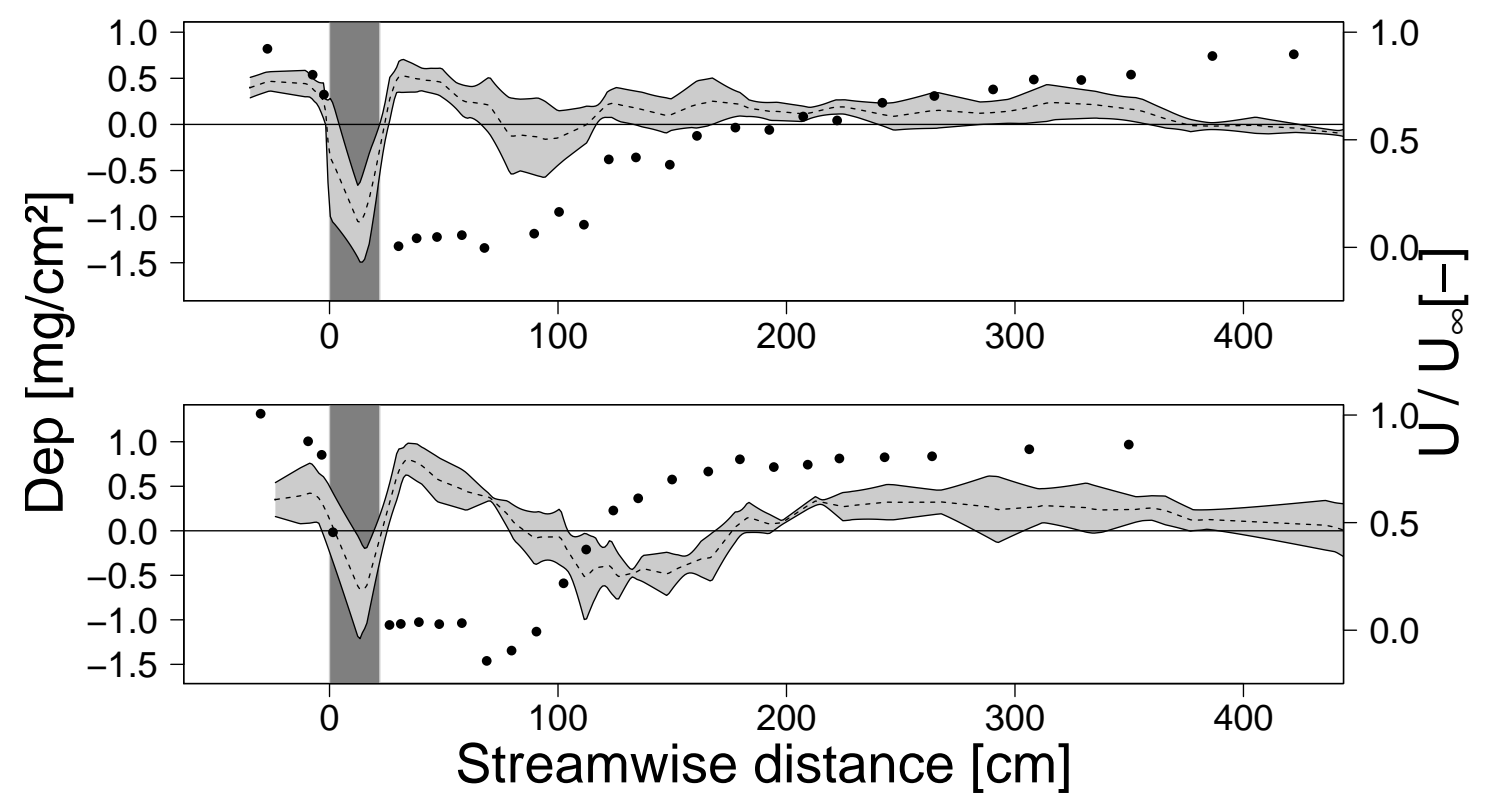

Figure 5: Average deposition $\left[\mathrm{mg} / \mathrm{cm}^{2}\right]$ from three measurements substracted from the mean deposition of each experimental run is shown in light gray, the interval indicating the error on the measurements. Mean, streamwise velocity $U$ on the profile through the center of the left patch, normalized by the upstream velocity $U_{\infty}$ for a gap spacing of $2 \mathrm{~cm}$ (above) and $11 \mathrm{~cm}$ (below) is indicated by solid, black dots. The position of the patch is indicated by a dark, grey bar. 
Flemish Research Organisation (FWO) for the Scientific Research Network (WOG): 'the functioning of river ecosystems through plant-flow-soil interactions'.

\section{REFERENCES}

Bouma, T. J., M. Friedrichs, B. K. van Wesenbeeck, S. Temmerman, G. Graf, \& P. M. J. Herman (2009, FEB). Density-dependent linkage of scale-dependent feedbacks: a flume study on the intertidal macrophyte Spartina anglica. OIKOS 118(2), 260-268.

Chen, Z., A. Ortiz, L. Zong, \& H. Nepf (2012, SEP 13). The wake structure behind a porous obstruction and its implications for deposition near a finite patch of emergent vegetation. WATER RESOURCES RESEARCH 48.

Cotton, J. A., G. Wharton, J. A. B. Bass, C. M. Heppell, \& R. S. Wotton (2006, JUL 30). The effects of seasonal changes to in-stream vegetation cover on patterns of flow and accumulation of sediment. GEOMORPHOLOGY 77(3-4), 320334. 100th Annual Meeting of the Association-of-AmericanGeographers, Philadelphia, PA, MAR 14-19, 2004.

Gurnell, A., G. Petts, D. Hannah, B. Smith, P. Edwards, J. Kollmann, J. Ward, \& K. Tockner (2001, JAN). Riparian vegetation and island formation along the gravel-bed Fiume Tagliamento, Italy. EARTH SURFACE PROCESSES AND LANDFORMS 26(1), 31-62.

Gurnell, A., K. Tockner, P. Edwards, \& G. Petts (2005, SEP). Effects of deposited wood on biocomplexity of river corridors. FRONTIERS IN ECOLOGY AND THE ENVIRONMENT 3(7), 377-382.

Jarvela, J. (2005, JUN 9). Effect of submerged flexible vegetation on flow structure and resistance. JOURNAL OF HYDROLOGY 307(1-4), 233-241.

Kouwen, N. \& T. Unny (1975). Flexible roughness in open channels. JOURNAL OF THE HYDRAULICS DIVISIONASCE 101(NHY1), 194-196.

Lee, J. H. \& V. Chu (2003). Turbulent jets and plumes: a Lagrangian Approach. Kluwer Academic Publishers.

McLelland, S. \& A. Nicholas (2000, FEB 15). A new method for evaluating errors in high-frequency ADV measurements. HYDROLOGICAL PROCESSES 14(2), 351-366.

Naden, P., P. Rameshwaran, O. Mountford, \& C. Robertson (2006, NOV 30). The influence of macrophyte growth, typical of eutrophic conditions, on river flow velocities and turbulence production. HYDROLOGICAL PROCESSES 20(18), 3915-3938.

Nepf, H. M. (2012). Flow and Transport in Regions with Aquatic Vegetation. In Davis, SH and Moin, P (Ed.), ANNUAL REVIEW OF FLUID MECHANICS, VOL 44, Volume 44 of Annual Review of Fluid Mechanics, pp. 123-142. 4139 EL CAMINO WAY, PO BOX 10139, PALO ALTO, CA 943030897 USA: ANNUAL REVIEWS.

Ortiz, A.and Ashton, A. \& H. Nepf (2014). Mean and turbulent velocity field near rigid and flexible plants, and the implications for deposition. Journal of Geophysical Research In Revision.

Rietkerk, M. \& J. Van de Koppel (2008, MAR). Regular pattern formation in real ecosystems. TRENDS IN ECOLOGY \& EVOLUTION 23(3), 169-175.

Sand-Jensen, K. (1998, JUN). Influence of submerged macrophytes on sediment composition and near-bed flow in lowland streams. FRESHWATER BIOLOGY 39(4), 663-679.

Schoelynck, J., T. De Groote, K. Bal, W. Vandenbruwaene, P. Meire, \& S. Temmerman (2012, AUG). Self-organised patchiness and scale-dependent bio-geomorphic feedbacks in aquatic river vegetation. ECOGRAPHY 35(8), 760-768.

Stephan, U. \& D. Gutknecht (2002, DEC 1). Hydraulic resistance of submerged flexible vegetation. JOURNAL OF HY-
DROLOGY 269(1-2), 27-43. 26th General Assembly of the European-Geophysical-Society, NICE, FRANCE, MAR 2630, 2001.

Sumner, D. (2010, AUG). Two circular cylinders in crossflow: A review. JOURNAL OF FLUIDS AND STRUCTURES 26(6), 849-899.

Sumner, D., S. Wong, S. Price, \& M. Paidoussis (1999, APR). Fluid behaviour of side-by-side circular cylinders in steady cross-flow. JOURNAL OF FLUIDS AND STRUCTURES 13(3), 309-338.

Temmerman, S., T. J. Bouma, J. Van de Koppel, D. Van der Wal, M. B. De Vries, \& P. M. J. Herman (2007, JUL). Vegetation causes channel erosion in a tidal landscape. $G E O L$ $O G Y$ 35(7), 631-634.

van de Koppel, J., M. Rietkerk, N. Dankers, \& P. Herman (2005, MAR). Scale-dependent feedback and regular spatial patterns in young mussel beds. AMERICAN NATURALIST 165(3), E66-E77.

van Wesenbeeck, B. K., J. van de Koppel, P. M. J. Herman, \& T. J. Bouma (2008, JAN). Does scale-dependent feedback explain spatial complexity in salt-marsh ecosystems? OIKOS 117(1), 152-159.

Vandenbruwaene, W., S. Temmerman, T. J. Bouma, P. C. Klaassen, M. B. de Vries, D. P. Callaghan, P. van Steeg, F. Dekker, L. A. van Duren, E. Martini, T. Balke, G. Biermans, J. Schoelynck, \& P. Meire (2011, FEB 16). Flow interaction with dynamic vegetation patches: Implications for biogeomorphic evolution of a tidal landscape. JOURNAL OF GEOPHYSICAL RESEARCH-EARTH SURFACE 116.

White, B. L. \& H. M. Nepf (2007, DEC 25). Shear instability and coherent structures in shallow flow adjacent to a porous layer. JOURNAL OF FLUID MECHANICS 593, 1-32.

Zong, L. \& H. Nepf (2010, APR 1). Flow and deposition in and around a finite patch of vegetation. GEOMORPHOL$O G Y$ 116(3-4, SI), 363-372. 40th Binghamton Geomorphology Symposium, Virginia Tech Blacksburg, Blacksburg, VA, OCT 02-04, 2009.

Zong, L. \& H. Nepf (2011, JAN 25). Vortex development behind a finite porous obstruction in a channel. JOURNAL OF FLUID MECHANICS 691, 368-391. 